\title{
TEXTURAL DESCRIPTORS FOR MULTIPHASIC ORE PARTICLES
}

\author{
LAURA PÉREZ-BARNUEVO $^{\varpi, 1}$, ERIC PIRARD ${ }^{2}$ AND RICARDO CASTROVIEJO ${ }^{1}$ \\ ${ }^{1}$ Universidad Politécnica de Madrid, Escuela de Ingenieros de Minas, C/ Ríos Rosas, 21, 28003 Madrid, Spain; \\ ${ }^{2}$ Université de Liège, GeMMe, Georesources and Geo-imaging Lab Sart Tilman B52, 4000 Liège, Belgium \\ e-mail: Laura.perez.barnuevo@upm.es; Ricardo.castroviejo@upm.es; Eric.pirard@ulg.ac.be \\ (Received July 30, 2012; revised October 23, 2012; accepted October 30, 2012)
}

\begin{abstract}
Monitoring of mineral processing circuits by means of particle liberation analysis through quantitative image analysis has become a routine technique within the last decades. Usually, liberation indices are computed as weight proportions, which is not informative enough when complex texture ores are treated by flotation. In these cases, liberation has to be computed as phase surface exposed to reactants, and textural relationships between minerals have to be characterized to determine the possibility of increasing exposure. In this paper, some indices to achieve a complete texture characterization have been developed in terms of 2D phase contact and mineral surfaces exposure. Indices suggested by other authors are also compared. The response of this set of parameters against textural changes has been explored on simple synthetic textures ranging from single to multiple inclusions and single to multiple veins and their ability to discriminate between different textural features is analyzed over real mineral particles with known internal structure.
\end{abstract}

Keywords: image analysis, linear intercepts method, mineral liberation, mineral processing, texture characterization.

\section{INTRODUCTION}

Ores are complex assemblages of mineral phases, some being economically valuable and others being considered as uneconomic, gangue material or even as penalties. The main objective of mineral processing is to separate the valuable fraction from the gangue material by making use of contrasted properties such as density, magnetic susceptibility, hydrophobicity, etc.

The most efficient separation techniques operate on monomineralic particles obtained after crushing and grinding, but obviously this ideal situation can hardly be achieved and most often multiphasic or socalled unliberated particles are present in the process. Usually, these composite particles are treated in a regrinding stage with the aim of producing liberated particles. However, size reduction not always leads to a significant increase in mineral liberation, because size is not the only factor that determines liberation: textural relationships between mineral phases that make up particles play an important role in the possibilities of achieving liberation. Hence, a complete characterization of particles should include not only mineral liberation quantification and size characterization, but also an adequate textural characterization, which is essential for mineral processing control, and consequently for increasing mineral recoveries.

\section{MINERAL CHARACTERIZATION}

In the last decades, sophisticated techniques for automatic mineral characterization have been developed based on the use of digital images provided by scanning electron microscopy or optical microscopy (Pirard et al., 2008, proposed multispectral imaging in the visible and near-IR realms; Castroviejo et al., 2010, applied it to a fully automated system). The use of these techniques allows for analyzing a huge quantity of particles and a great variety of mineral properties, which would be impossible to measure accurately by manual methods.

Mineralogical analysis performed by automatic techniques includes qualitative and quantitative data about composition, grain size and mineral liberation, which is one of the most useful parameters for metallurgists. Liberation can be expressed in different ways, being weight proportion the most frequently used. However, when it deals with flotation, liberation must also be expressed in terms of exposed surface proportion. Nevertheless, when complex textures are present in the ore, both values of liberation differ (Lastra, 2002) and a complete characterization of texture is required to establish the possibility of liberation by physical or chemical means. 
According to the definition of mineral texture given by Ramdohr (1980) in relation to mineral dressing, the main textural features that should be quantified are grain size and intergrowth relationships, including the number and nature of boundaries and the type of intergrowth between ore and gangue minerals. While grain size and grade of contact are usual outputs of mineralogical analysis, automatic characterization of intergrowth type has not been resolved yet, despite having been pointed out by many authors (Gaudin, 1939; Amstutz and Giger, 1972; Craig and Vaughan, 1994) as essential to avoid unnecessary costs and to optimize the process.

With the aim of automatically identifying the four types of intergrowth considered as the most relevant from the point of view of particles behavior during flotation (Fig. 1) and providing a more complete characterization of mineral particles, some textural descriptors have been developed. Additionally, various indices for the quantification of the grade of contact between phases proposed by other authors are explored.

\section{MATERIALS AND METHODS}

The method to characterize textural features presented here is intended to be applied on digital images provided by scanning electron microscopy or optical microscopy. In both cases, mineral particles are mounted on polished sections, whose surfaces are scanned and a number of particles, large enough to guaranty the sample representativeness, is taken for a later analysis. After acquisition, the images have to be processed to identify and classify every phase: ore and gangue minerals and also the background or mounting media. In the final image, every single grain is classified and has a homogeneous grey level, so boundaries between phases are well defined. After this process, each mineral particle in the image is individually analyzed by the linear intercepts method, which provides a set of measurements to obtain several parameters, both geometric and stereological. Based on these parameters some indices are computed and their ability to discriminate different textural features in real mineral particles is tested by discriminant analysis. Moreover, the metallurgical meaning of each index is analyzed.

Although the final aim of this research is working with real mineral particles, first attempts of texture characterization have been carried out on a series of textures in synthetic biphasic particles that represent the intergrowths described by Gaudin (1939) in a simple way (Fig. 2). In these particles, the proportion of each phase is fixed (60\% of dark phase and $40 \%$ of bright phase) and the internal structure varies from single to branching veins (simple to stockwork intergrowth, cases 1 to 5) and from single to multiple inclusions randomly distributed (coated intergrowth to emulsion-like intergrowth, cases 6 to 10). The first five cases of Fig. 2 have been drawn with MATLAB, by means of a program that randomly generates a certain number of Poisson bands of known maximum width inside a circle of known diameter. Cases 6 to 10 have been drawn with a photo editor program (Corel PaintShop Pro), generating circles of known diameter. The main advantage of these particles is their known geometry, which enables to check results by comparison with the analytical solution.

Working with biphasic particles may seem an oversimplification, but it is a realistic approach for textural analysis. Following this approach, mineral particles can be treated as biphasic, if we want to identify which is the type of intergrowth of one specific phase within the particle. In this case, one phase is the phase of interest (POI from now on) designated by $\alpha$, and the other phase (designated by $\beta$ ) comprises the remaining constituents of the particle.
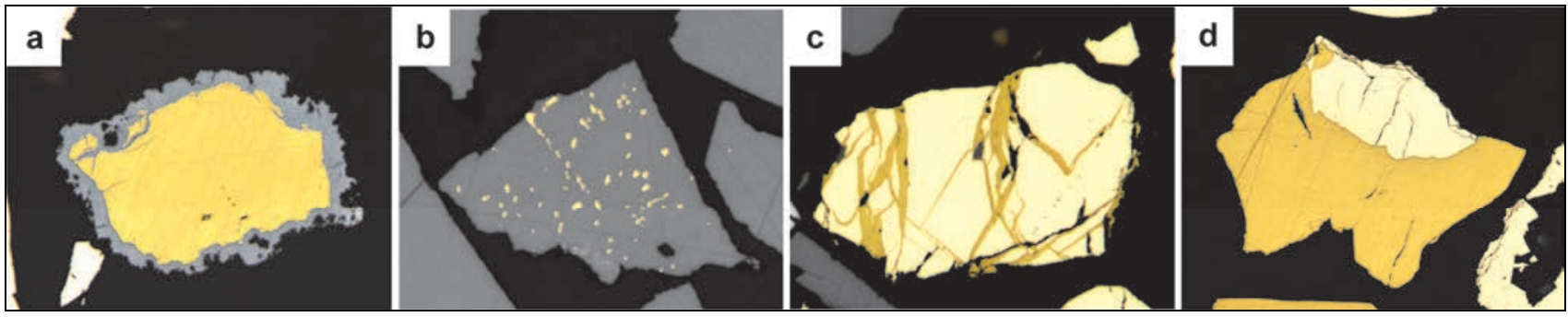

Fig. 1. Real ore particles to illustrate typical intergrowths described by Gaudin as the most relevant in flotation (a: coated; b: emulsion; c: stockwork; d: simple). 


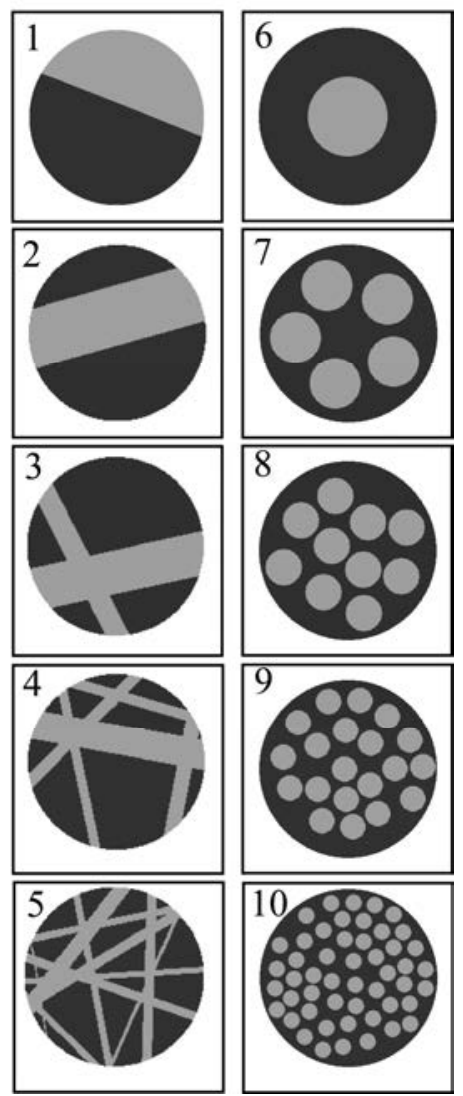

Fig. 2. Synthetic particles: from simple to branching veins (1 to 5) and from one inclusion to multiple inclusions (6 to 10).

\section{LINEAR INTERCEPTS METHOD}

The linear intercepts method has been chosen because it enables the quantification of specific mineralogical features, like phase area and perimeter, or grain surface area per unit volume, through simple stereological relationships based on simple counts and measurements. The quantification of these parameters, as shown later, determines directly some aspects of the metallurgical behavior of the ore. However, its great potential for texture characterization is not only the quantification of these parameters, but also the generation of linear liberation grade distributions, which is considered a function of texture (Jones et al., 1978; Jones and Horton, 1979; Schneider, 1995).

In this case, the method is applied by superimposing a set of parallel lines on the particle, separated from each other with an equidistance of 1 pixel. Along each test line the number of intersections between the line and each grain boundary is counted, identifying the type of boundary intercepted as a phase-to-phase contact or a phase-to-background contact (Fig. 3a). In addition to the number of intersections, the length of the test line upon each phase in the particle is measured (Fig. 3b).
Once every line of the probe has been analyzed, both the total length of line across each phase and the total number of intersections of each type are obtained by adding up the measurements made across each test line.

This procedure is carried out for 18 different orientations, rotating the particle at regular angular intervals of $10^{\circ}$ in order to characterize its internal structure, satisfying for the sample the conditions of isotropy, uniformity and randomness (IUR) (Russ and DeHoff, 2000) necessary for unbiased estimations.

Parameters computed with the counts and measurements made in the 18 directions are described below.

\section{GEOMETRIC AND STEREOLOGICAL PARAMETERS}

There are a large number of parameters derived from direct measurements made by the linear intercepts method or calculated from them. In this work, three of these parameters have a special significance for the characterization of mineral phases within particles: phase area, phase perimeter and surface density of phase contact interfaces.

\section{Phase area}

The area of one phase is easily computed as the sum of every pixel in the image belonging to that phase, multiplied by the corresponding calibration coefficient. In this case, as the linear intercept method is being applied with an equidistance of 1 pixel between line probes, the total number of pixels belonging to the POI is equal to the total length of all test lines across it. The area of the POI is given by Eq. 1 (where $\mathrm{c}$ is the calibration coefficient).

$$
A(\alpha)=\sum L_{i}^{\theta}(\alpha) \times c .
$$

This equation, as shown later, will be used to get the volumetric fraction of each phase, $\mathrm{VV}$, by the wellknown stereological principle given by Eq. 2 (Underwood, 1970).

$$
V_{V}=A_{A}=L_{L}=P_{P} .
$$

\section{Phase perimeter}

Perimeter fractions are essential for the characterization of mineral liberation, especially when a complex texture exists. There are a number of ways for perimeter computation based on pixel operations (Russ, 1990), but these methods are less accurate than the estimation of the perimeter from the number of intersections. 
For this purpose, Barbier's formula (Eq. 3), whose derivation is beyond the scope of this work (see Hyksova et al., 2012, for further information), is applied for length estimation of curves in plane by counting the number of intersections of the curve with a system of line probes in different directions:

$$
L=\frac{\pi d}{2} \times \bar{N}
$$

being $d$ the distance between test lines and the mean number of intersections of the curve with test lines for all line orientations.

In the example of Fig. 3, this formula has been applied for the estimation of the $\alpha \beta$ contact perimeter using the intercept method as described in this paper. Using the notation in Fig. 3, the perimeter of the $\alpha \beta$ contact will be calculated as follows:

$$
B(\alpha \beta)=\frac{\pi d}{2} \times \overline{\sum_{\theta} \sum_{i} N_{i}^{\theta}(\alpha \beta)} .
$$

\section{Specific surface area $\left(\mathrm{S}_{\mathrm{V}}\right)$}

The surface area per unit volume $\left(\mathrm{S}_{\mathrm{V}}\right)$ is especially useful as we are concerned with the determination of contacts between phases within particles. This parameter is easily calculated from the length of grain boundaries per unit area (LA) or from the number of intersections per unit length of test line (PL) applying one of these stereological relationships (Underwood, 1970):

$$
\begin{aligned}
& S_{v}=\frac{4}{\pi} \times L_{A}, \\
& S_{v}=2 \times P_{L} .
\end{aligned}
$$

Following with our example in Fig. 3, the mean surface area density of the $\alpha \beta$ contact is given by Eq. 7 or 8 . In Eq. 7 length of grain boundaries $B(\alpha \beta)$ is got applying Eq. 4:

$$
\begin{gathered}
S_{V}(\alpha \beta)=\frac{4}{\pi} \times \frac{B(\alpha \beta)}{A}, \\
S_{V}(\alpha \beta)=2 \times \frac{\frac{\sum_{\theta} \sum_{i} N_{i}^{\theta}(\alpha \beta)}{\sum_{i} L_{i}^{\theta}} .}{} .
\end{gathered}
$$

\section{LINEAR LIBERATION GRADES DISTRIBUTION}

For each test line, linear liberation is calculated as the proportion of phase $\alpha$ across the line (Eq. 9).

$$
L_{L i}^{\theta}(\alpha)=\frac{L_{i}^{\theta}(\alpha)}{L_{i}^{\theta}} .
$$

If the line is composed just by this phase it is considered as liberated, and the total phase area on liberated test lines is called apparent linear liberation. In contrast, if there is more than one phase, the line is considered as non-liberated and the total amount of phase in non-liberated test lines is added to get the average linear liberation and to generate the distribution of linear liberation grades. This distribution has been analyzed to get valuable information for texture characterization.

Besides this, the apparent linear liberation is also intimately related to texture by this simple observation: the more complex the particle texture, the lower its apparent linear liberation, because the probability of finding a liberated test line (i.e., one which does not cross any $\alpha \beta$ boundary) decreases as the surface density of the $\alpha \beta$ interface increases.

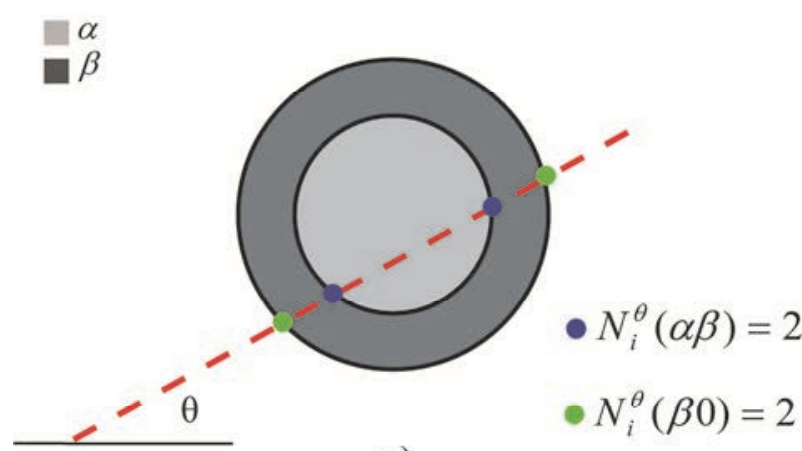

a)

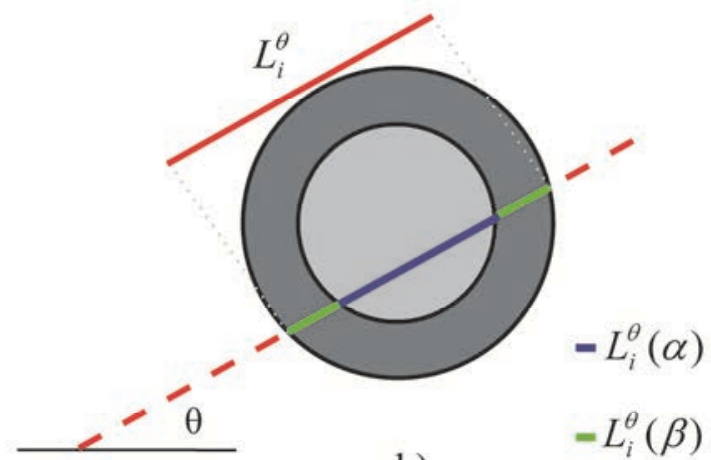

b)

Fig. 3. Linear Intercepts Method applied to the measurement of the number of intersections (a) and the length of test line across each particle's component (b) for the ith line in direction $\theta$. 


\section{TEXTURAL DESCRIPTORS DEFINITION}

Based on the parameters described above, some indices have been developed with two main purposes: to quantify the most relevant mineralogical features for mineral processing, and to provide textural information discriminating between different types of intergrowth. Some of these indices satisfy both conditions, while others (like those computed from linear liberation) have been developed because of their expected discriminating power. Along with these descriptors, the most relevant intergrowth indices proposed by other authors (Gurland, 1958; Amstutz and Giger, 1972; Jeulin, 1981) are analyzed.

Before describing textural descriptors it is convenient to make some comments on the notations used for their formulation. As mentioned before, the phase of interest is represented by $\alpha$ while the remaining phases in the particle are named with $\beta, \gamma, \delta$. To refer to all the phases in the particle $\varphi$ is used as a generic symbol, 0 represents the background (resin in real sections) and finally, - represents all phases, including background. Founded on this notation, indices are formulated for the POI's $(\alpha)$ and they are classified according to their metallurgical significance and origin.

\section{CLASSICAL LIBERATION INDICES}

Usually, mineral liberation is expressed by two different parameters: weight ratio and exposure ratio. In single particles, the former indicates the weight proportion of one mineral with regard to the total particle weight, while the latter quantifies the proportion of exposed perimeter occupied by this mineral. Both of them are essential for the characterization of mineral particles, because they indicate the value of the particle and the possibility for recovery by flotation, respectively. Weight proportion is obtained from Eq. 10 multiplying by mineral density, while exposed perimeter proportion is derived from Eq. 11, dividing exposed perimeter of $\alpha$ by total exposed perimeter of the particle. Both perimeters are calculated using Barbier's formula and the notation used is explained in Fig. 3.

$$
\begin{gathered}
A_{A}(\alpha)=\frac{\sum_{i} L_{i}^{\theta}(\alpha)}{\sum_{i} L_{i}^{\theta}(\varphi)}, \\
B_{B}(\alpha)=\frac{\sum_{\theta} \sum_{i} N_{i}^{\theta}(\alpha, o)}{\sum_{\theta} \sum_{i} N_{i}^{\theta}(\varphi, o)} .
\end{gathered}
$$

\section{PHASE CONTACT INDICES}

This group of indices quantifies the degree of contact between phases within particles by means of different $S_{V}$ ratios. In this group, the indices proposed by other authors (Gurland, 1958 (Eq. 15); Amstutz and Giger, 1972 (Eq. 13); Jeulin, 1981 (Eq. 14)) are included, after being adapted to the special case of particleby-particle analysis instead of analyzing a multiphase material aggregate. Along with them, an additional index (Eq. 12) has been developed. For each index, both formulae based on surface area density $\left(\mathrm{S}_{\mathrm{V}}\right)$ and its simplification based on intercept counts (Eq. 8) are shown.

Phase contact index. Ratio between the surface density of the $\alpha \beta$ intergrowth surface and the surface density of the particle exposed surface.

$$
I_{C}(\alpha \beta)=\frac{S_{V}(\alpha \beta)}{S_{V}(\varphi \circ)}=\frac{\sum_{i} N_{i}^{\theta}(\alpha \beta)}{\sum_{i} N_{i}^{\theta}(\varphi o)}
$$

Intergrowth index (Amstutz and Giger, 1972). The so-called intergrowth or locking index is a numerical value which indicates how much of each phase is intergrown with all other phases. It is a ratio between the surface density of the intergrowth surface between two phases and the total surface density in the particle.

$$
I_{A-G}(\alpha \beta)=\frac{S_{V}(\alpha \beta)}{S_{V}(\varphi \bullet)}=\frac{\sum_{i} N_{i}^{\theta}(\alpha \beta)}{\sum_{i} N_{i}^{\theta}(\varphi \bullet)} .
$$

Coordination index (Jeulin, 1981). Indicates the probability of the $\alpha \beta$ contact in a multiphasic system.

$$
\begin{aligned}
I_{J}(\alpha \beta)= & \frac{S_{V}(\alpha \beta) \times S_{V}(\varphi \bullet)}{S_{V}(\alpha \bullet) \times S_{V}(\beta \bullet)}= \\
= & \frac{\sum_{i} N_{i}^{\theta}(\alpha \beta) \times \sum_{i} N_{i}^{\theta}(\varphi \bullet)}{\sum_{i} N_{i}^{\theta}(\alpha \bullet) \times \sum_{i} N_{i}^{\theta}(\beta \bullet)}
\end{aligned}
$$

Contiguity Index (Gurland, 1958). Originally this index was defined to compute the fraction of the total interface area of a phase that is shared by particles of the same phase. Its derivation for multiphase systems is given in Eq. 15.

$$
I_{C G}(\alpha \beta)=\frac{S_{V}(\alpha \beta)}{S_{V}(\alpha \bullet)}=\frac{\sum_{i} N_{i}^{\theta}(\alpha \beta)}{\sum_{i} N_{i}^{\theta}(\alpha \bullet)}
$$

\section{TEXTURE COMPLEXITY INDEX (ITC)}

One last index has been developed based on intersection counts to give an idea of the texture complexity. 
In a biphasic particle, the minimum surface density possible for the intergrowth surface between two phases is estimated from the number of biphasic test lines applying Eq. 6 (e.g. in the simplest case there is just one intersection of each test line with the $\alpha \beta$ contact). On the other hand, the real surface density of the intergrowth surface can be computed from the number of real intersections between test lines and the $\alpha \beta$ boundary (Eq. 6). The ratio between these two surface densities is an expression of the texture complexity and grain boundary irregularity: values close to unity belong to the simplest intergrowth, while the higher values indicate the existence of complex texture. Table 1 contains $\mathrm{I}_{\mathrm{TC}}$ values for synthetic particles in Fig. 2. As shown in this table, the more complex the $\alpha \beta$ contact, the higher the value of this index, because the probability of intersecting the $\alpha \beta$ boundary by the same test line increases with texture complexity.

$$
I_{T C}=\frac{\mathrm{S}_{\mathrm{V}}(\alpha \beta)}{S_{V} \min (\alpha \beta)}=\frac{\sum_{i} N_{i}^{\theta}(\alpha \beta)}{\sum_{\theta} I_{i}^{\theta}} \text { iff } L_{L i}^{\theta}(\alpha) \neq 1,
$$

where $\sum_{\theta} I_{i}^{\theta}$ represents the total number of biphasic test lines.

\section{LINEAR LIBERATION INDICES}

Two indices are directly derived from the computation of linear liberation: the average linear liberation (Eq. 17) and the apparent linear liberation (Eq. 18). Moreover, the shape of the distribution of linear liberation grades is analyzed to get another two indices, computed from the percentiles $\mathrm{P}_{10}, \mathrm{P}_{50}$ and $\mathrm{P}_{90}$ of this distribution (Eqs. 19, 20).

$$
\begin{gathered}
\overline{L_{L}}(\alpha)=\frac{\sum_{\theta} L_{i}^{\theta}(\alpha)}{\sum_{\theta} L_{i}^{\theta}} \text { iff } L_{L i}^{\theta}(\alpha) \neq 1, \\
A_{L L}(\alpha)=\frac{\sum_{\theta} L_{i}^{\theta}(\alpha)}{\sum_{\theta} L_{i}^{\theta}} \text { iff } L_{L i}^{\theta}(\alpha)=1, \\
L_{L 1}(\alpha)=\frac{P_{90}-P_{50}}{P_{90}-P_{10}},
\end{gathered}
$$

$$
L_{L 2}(\alpha)=\frac{P_{90}-P_{50}}{P_{50}-P_{10}}
$$

\section{TEXTURAL DESCRIPTORS ANALYSIS}

Textural descriptors given by Eqs. 10-20 have been computed for the bright phase ( $\alpha$ phase) in the synthetic biphasic particles of Fig. 2. The response of these indices to textural variations and their ability to discriminate the intergrowth type is analyzed by discriminant analysis. On the other hand, their metallurgical meaning is also highlighted as they quantify significant mineralogical features, like particle composition, grade of locking, degree of contact between phases and grain boundary irregularity.

\section{TEXTURAL DESCRIPTORS AS TEXTURE IDENTIFIERS}

Textural descriptors have been represented against texture complexity index ( $\mathrm{I}_{\mathrm{TC}}$, Eq. 16) in order to analyze their response to textural variations. In the next series of figures, textural descriptors computed for the bright phase in particles ranging from simple texture to stockwork (left graph) and from coated to emulsion (right graph) are represented in ordinates against $\mathrm{I}_{\mathrm{TC}}$ (in abscissa). In Fig. 4a index $\mathrm{I}_{\mathrm{C}}$ (Eq. 12) is represented in ordinates; in Fig. 4b the indices proposed by other authors ( $\mathrm{I}_{\mathrm{A}-\mathrm{G}}$ (Eq. 13), $\mathrm{I}_{\mathrm{J}}$ (Eq. 14) and $\mathrm{I}_{\mathrm{CG}}$ (Eq. 15)) are represented in ordinates to test their evolution against texture complexity. Finally, in Fig. 4c the response to texture complexity of indices derived from the distribution of linear liberation is analyzed.

As shown in Fig. 4a index Ic (Eq. 12) evolves linearly with texture complexity. Also indices proposed by other authors $\left(\mathrm{I}_{\mathrm{A}-\mathrm{G}}, \mathrm{I}_{\mathrm{CG}}\right.$ and $\mathrm{I}_{\mathrm{J}}$ ) show variations with an increase in texture complexity (Fig. 4a left). Moreover, when locking exists $\mathrm{I}_{\mathrm{CG}}$ and $\mathrm{I}_{\mathrm{J}}$ take a constant value (Fig. 4b right). Indices derived from linear liberation grades distribution do not seem to change with texture variations (Fig. 4c). However, their general trend seems to be characteristic for each series of particles.

Table 1. $I_{T C}$ values for synthetic particles in Fig. 2.

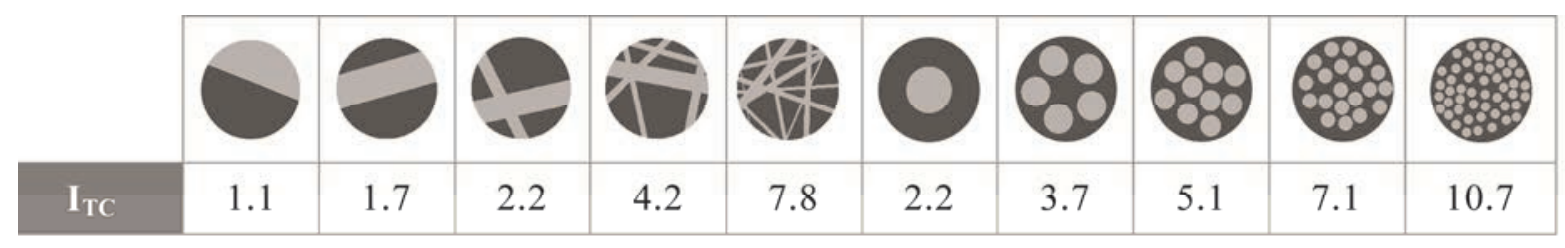




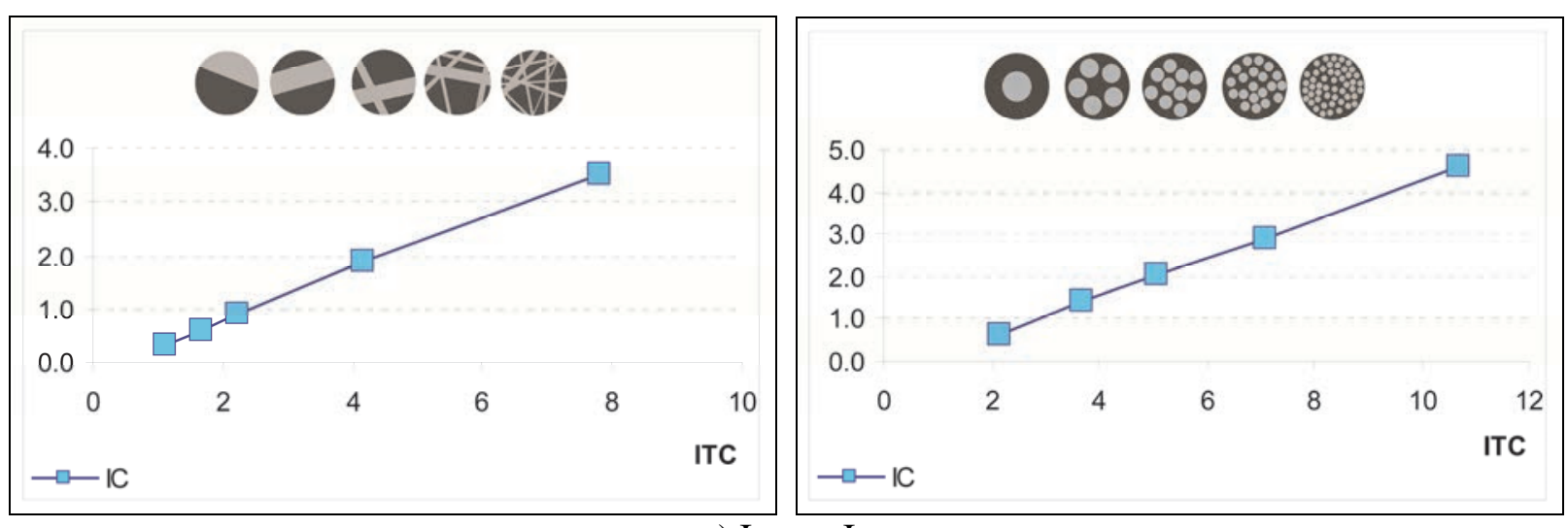

a) $I_{T C}$ Vs. $I_{C}$

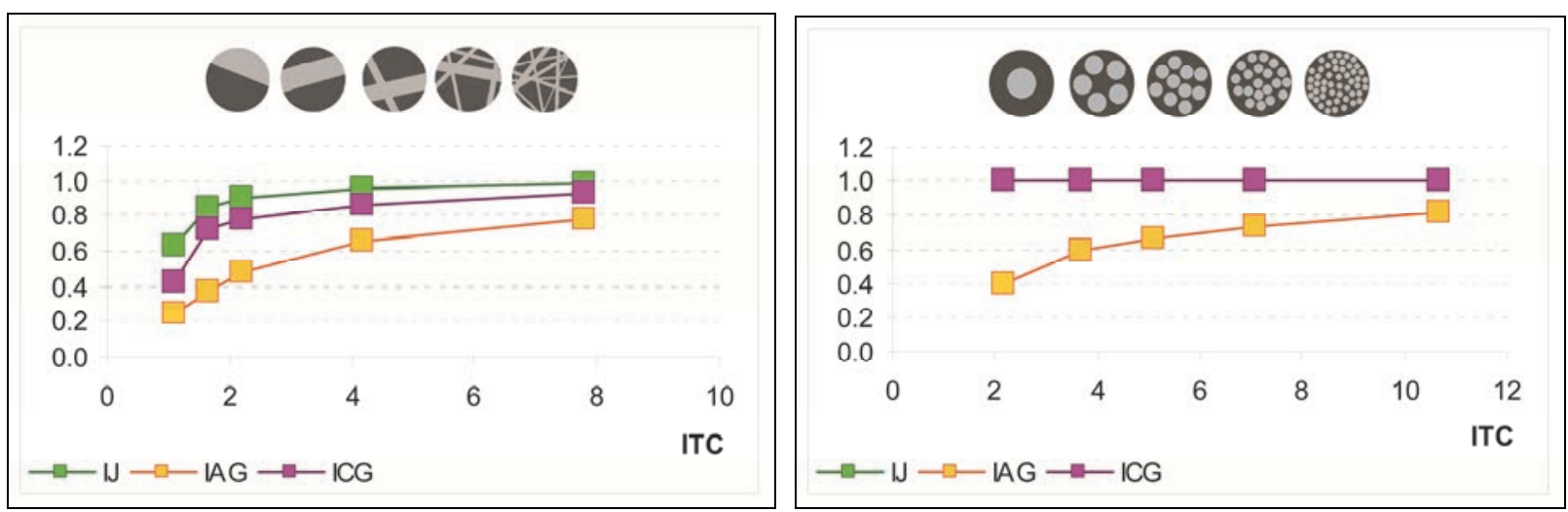

b) $\mathrm{I}_{\mathrm{TC}}$ Vs. $\mathrm{I}_{\mathrm{J}}-\mathrm{I}_{\mathrm{A}-\mathrm{G}}-\mathrm{I}_{\mathrm{CG}}$

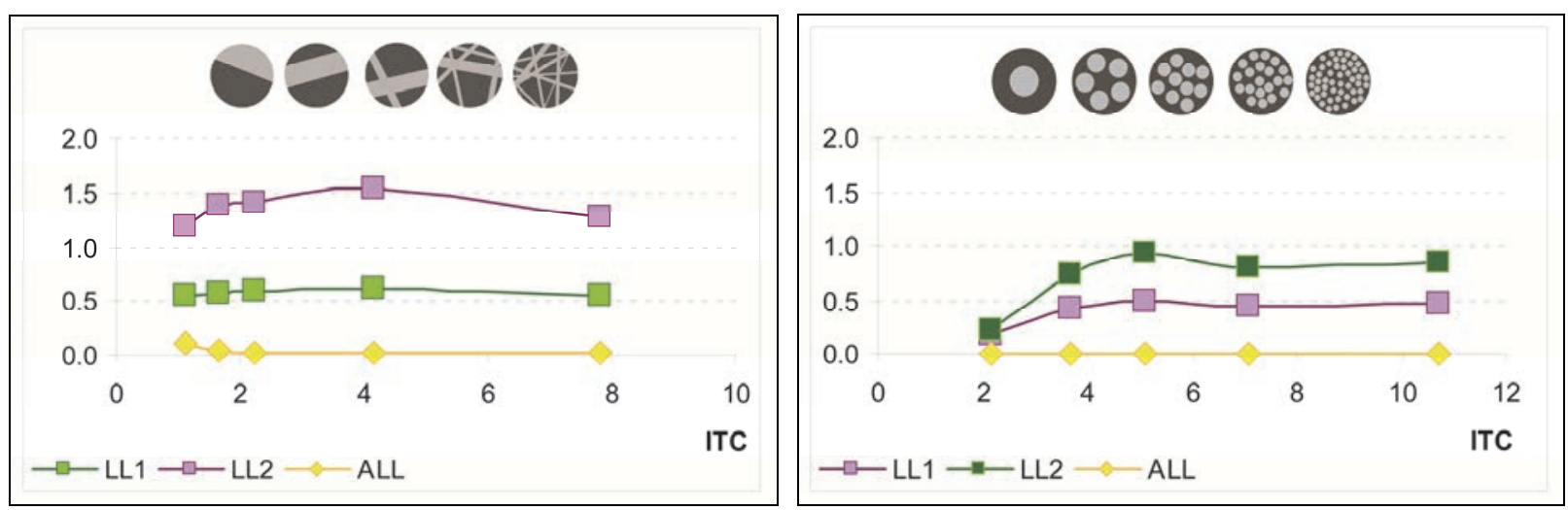

c) $\mathrm{I}_{\mathrm{TC}}$ Vs. $\mathrm{L}_{\mathrm{L} 1}-\mathrm{L}_{\mathrm{L} 2}-\mathrm{A}_{\mathrm{LL}}$

Fig. 4. Evolution of textural descriptors (ordinates) against texture complexity Index ( $I_{T C}$, abscissas) for the bright phase in particles ranging from simple texture to stockwork (left column), and from coated to emulsion (right column). In Fig $4 b$-right, curves for $I_{J}$ and $I_{C G}$ are superimposed.

This behavior against texture changes indicates that most of the proposed indices might be used to distinguish between different types of intergrowth. To establish which ones are the most effective a discriminant analysis has been carried out over a total of 77 real mineral particles from different samples. These particles have been selected according to their internal structure, so that one of the types of intergrowth shown in Fig. 2 can be easily recognized in any of their constituents. Some examples of these particles are shown in
Fig. 5. As they are processed as biphasic particles, the initial four types of intergrowths are in fact seven possible types. Thus, in particles belonging to type $a$, the phase of interest may be in the form of a single inclusion coated by a rim or it may be the rim itself. In the case of particles type $b$, the POI may be the emulsion or the matrix that contains the emulsion and the same can be said about particles of type $c$. Finally, the intergrowth in particles of type $d$ is considered as simple. Working with biphasic particles also implies 
that for each particle there are two possible intergrowths (one for each phase), so the initial 77 cases considered are actually 154 cases to be processed by discriminant analysis.

For both phases present in the particles, indices in Eqs. 10-20 were computed and correlations among them were evaluated in order to select the most efficient ones and to limit redundancy in the variables. The correlation matrix shows that a strong correlation exists between $\mathrm{I}_{\mathrm{C}}$ and $\mathrm{I}_{\mathrm{TC}}, \mathrm{I}_{\mathrm{C}}$ and $\mathrm{I}_{\mathrm{A}-\mathrm{G}}, \mathrm{L}_{\mathrm{L} 1}$ and $\mathrm{L}_{\mathrm{L} 2}$. Therefore, in each pair, the index with the lowest discriminant power was discarded. This analysis has been carried out with a discriminant function analysis and the classification is performed by the minimum Mahalanobis distance criteria. Results show that 95.5\% cases were correctly classified using the following four indices: $\mathrm{I}_{\mathrm{J}}, \mathrm{I}_{\mathrm{CG}}, \mathrm{I}_{\mathrm{TC}}$ and $\mathrm{L}_{\mathrm{L} 1}$. As shown in Table 2, phases belonging to the groups simple, emulsion and emulsion matrix were $100 \%$ correctly classified. Incorrectly classified cases are shown in Table 3.

Table 2. Classification results

\begin{tabular}{lc}
\hline & \% Correct \\
\hline Simple & 100.0 \\
Stockwork Matrix & 86.7 \\
Stockwork & 80.0 \\
Emulsion & 100.0 \\
Emulsion Matrix & 100.0 \\
Inclusion & 96.2 \\
Rim & 96.2 \\
\hline Total & 95.5 \\
\hline
\end{tabular}

Each row in this table contains a particle in which the inter-growth type of at least one of the phases has been incorrectly classified. For each phase (bright and dark phase) the observed intergrowth type (OC) and the intergrowth type given by discriminant analysis (DAC) are shown. Moreover, the Mahalanobis distance to the group into which each phase has been classified (DAD) and the Mahalanobis distance to the group into which the phase should have been classified (OCD) are also given. As shown in Table 3 the higher error occurs for the stockwork and stockwork matrix intergrowth types. However, for these intergrowth types, little difference exists between Mahalanobis distance to the observed classification group and Mahalanobis distance to de discriminant analysis classification group.

\section{TEXTURAL DESCRIPTORS AS MINERAL PROCESSING INDICATORS}

Although the main objective of these indices is the discrimination between different types of mineral intergrowths, the assessment of their mineralurgical meaning is also important. The first two indices that have to be computed are $A_{A}$ and $B_{B}$. The first one quantifies the amount of phase borne by each particle, which for real mineral particles means mineralogical value. However, a particle that carries the POI but has very low surface exposure to reactants (low $B_{B}$ ) would not be recovered by flotation in spite of having a high mineralogical value. So, both of these indices have to be computed and if they differ, a textural analysis will be required to establish the possibilities of acting on the particle in order to increase its liberation.

In addition to $\mathrm{B}_{\mathrm{B}}$, there are two more indices that show evidence of locking: $\mathrm{I}_{\mathrm{CG}}$ and $\mathrm{I}_{\mathrm{J}}$. Both of them are equal to 1 when there is a locked phase in a biphasic particle. However, while the first one is calculated for each phase in the particle and specifies which phase is included, the second one just indicates the existence of locking in the particle. For those cases in which a high degree of locking has been detected by one of the three indices $\left(\mathrm{B}_{\mathrm{B}}, \mathrm{I}_{\mathrm{CG}}, \mathrm{I}_{\mathrm{J}}\right)$ the degree of intergrowth and the intergrown surface irregularity has to be analyzed in order to assess the possibility of increasing liberation. The intergrowth index proposed by Amstutz and Giger (1972) (Eq. 13) is a good estimator of the former, while the latter can be estimated by the $\mathrm{I}_{\mathrm{TC}}$ (Eq. 16).

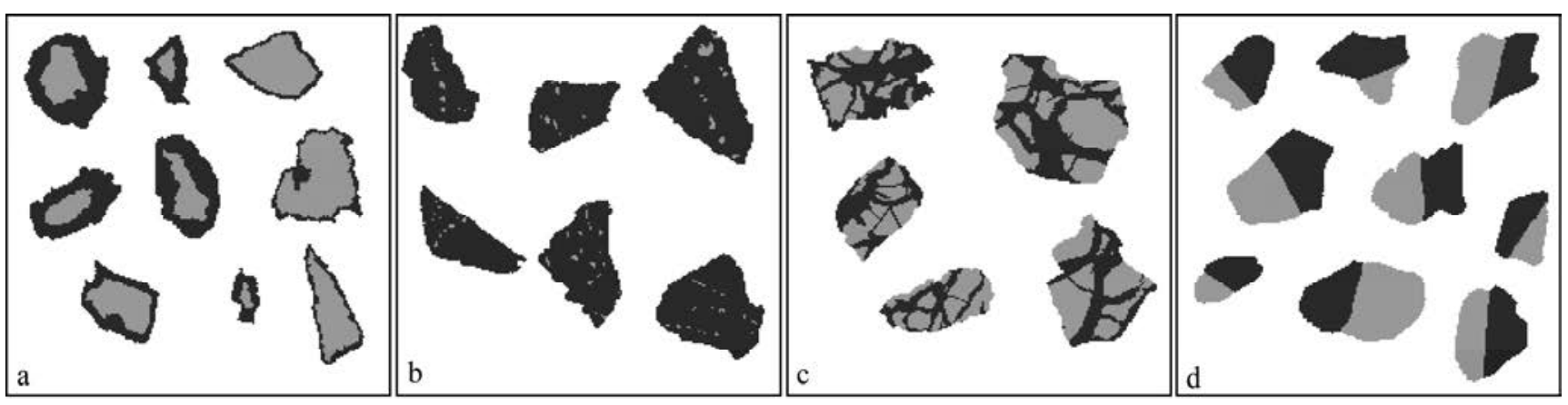

Fig. 5. Some examples of real particles used for discriminant analysis. 
Table 3. Incorrectly classified cases. OC: Observed classification (real intergrowth type). OCD: Mahalanobis distance to the observed classification group. DAC: Discriminant Analysis classification (intergrowth type given by discriminant analysis). DAD: Mahalanobis distance to the discriminant analysis classification group.

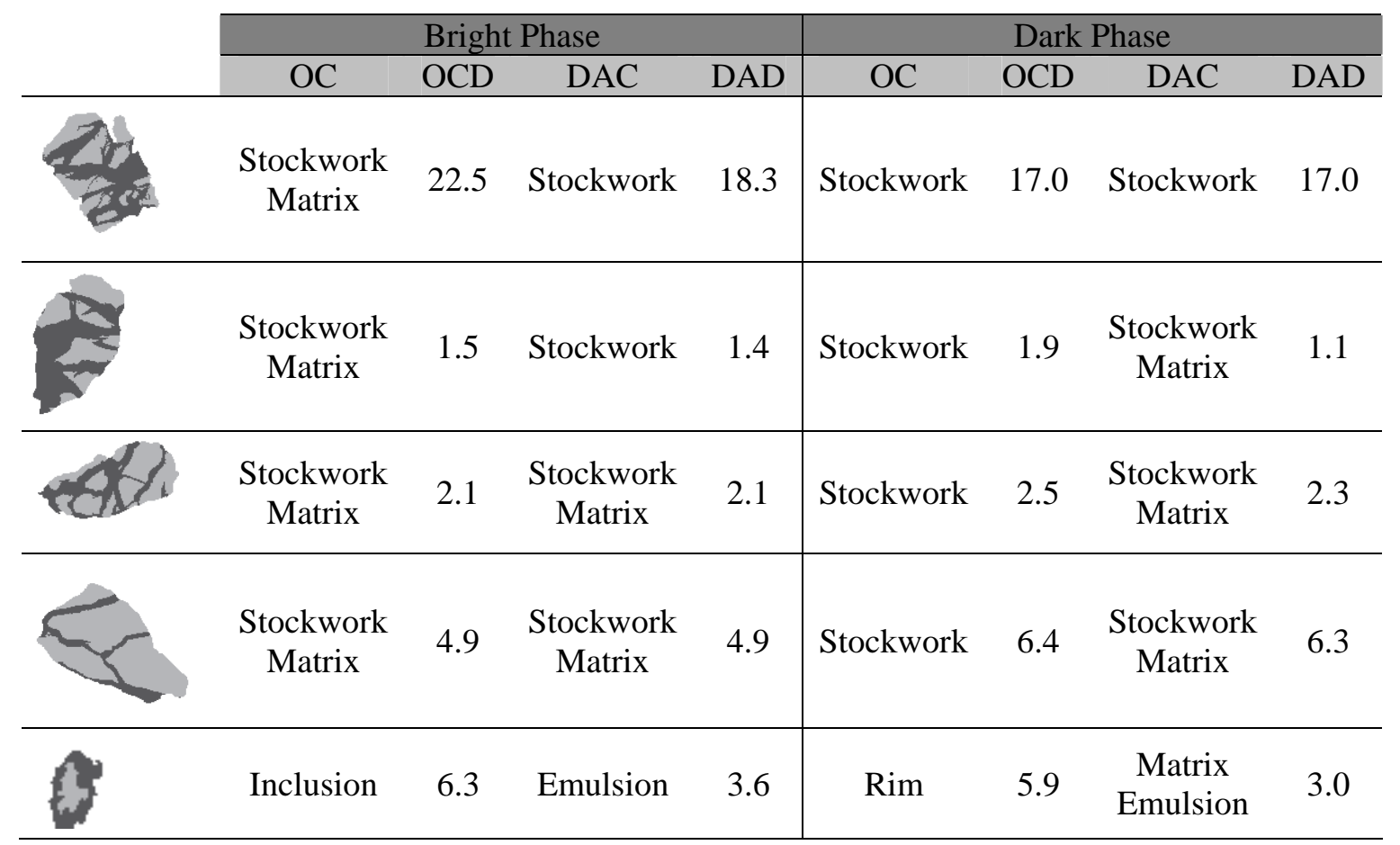

\section{CONCLUSIONS}

The automated characterization of the intergrowth type of mineral phases within mineral particles is possible using the new methodology proposed. This methodology is supported by a set of descriptors based on stereological parameters, which have been developed using the linear intercepts method. Comparison with some indices proposed by other authors to quantify the type of contact between mineral phases shows that the efficiency of these as discriminant variables is improved if complemented with the descriptors proposed in this work. Furthermore, assessment of their mineralurgical meaning shows their potential for practical application to geometallurgical purposes.

Discriminant analysis results show the capability of these indices to classify mineral phases according to their intergrowth type. This analysis shows that 95.5\% of cases analyzed were correctly classified using four indices: $\mathrm{I}_{\mathrm{J}}, \mathrm{I}_{\mathrm{CG}}, \mathrm{I}_{\mathrm{TC}}$ and $\mathrm{L}_{\mathrm{L} 1}$. It also indicates that three mineralogical parameters appear as significant for texture identification: locking grade (quantified by indices proposed by Gurland (1958) and Jeulin (1981)), texture complexity (quantified by $\mathrm{I}_{\mathrm{TC}}$, this work) and the shape of linear liberation grades distribution (expressed with index $\mathrm{L}_{\mathrm{L} 1}$, this work). The $95.5 \%$ success rate found appears as reasonable, taking into account that the morphology of complex intergrowths shows features that may be transitional to different types and tends to be ambiguous even for a human observer.

The results discussed in this paper show the potential of the proposed methodology to discriminate between the intergrowth types considered and to provide the mineralogical information required to achieve a complete mineralogical characterization regarding particles behavior during mineral processes. However, further tests have to be performed in order to improve the classification and to extend the methodology for the discrimination between new types of intergrowth. The tests performed suggest that, in some cases, textural analysis may require the use of different parameters to optimize the results for particular textures.

\section{ACKNOWLEDGEMENTS}

This research was supported by a FPI Grant from Universidad Politécnica de Madrid and by Project CAMEVA (CGL2006-13688-C02-01), funded by the Spanish Research Authority (Ministerio de Educación y Ciencia, Madrid). The contribution of Dr. Ida Eržen, Editor in Chief of the review, and two anonymous reviewers to the improvement of the original text is gratefully acknowledged. 


\section{REFERENCES}

Amstutz GC, Giger H (1972). Stereological methods applied to mineralogy, petrology, mineral deposits and ceramics. J Microsc 25: 145-64.

Castroviejo R, Catalina JC, Bernhardt HJ, Pirard E, Segundo F, Brea C, Pérez-Barnuevo L (2010). A fully automated system for multispectral ore microscopy. 20th General Meeting of the International Mineralogical Association, 2010 August 21-27, Budapest (Hungary), p. 291.

Craig J, Vaughan D (1994). Ore microscopy \& ore petrography. Editorial John Wiley and sons, Inc. Second Edition. New York - United States of America. 434.

Gaudin AM (1939). Principles of mineral dressing. New York: McGraw-Hill.

Gurland J (1958). The measurement of grain contiguity in two-phase alloys. Trans Am Inst Mining Met Engrs 212: 452-547.

Hyksova M, Kalousova A, Saxl I (2012). Early history of geometric probability and stereology. Image Anal Stereol 31: $1-16$.

Jeulin D (1981). Mathematical morphology and multiphase materials. Proc. $3^{\text {rd }}$ European Symposium on Stereology, Ljubljana, p. 265-86.

Jones MP, Horton R (1979). Recent developments in the stereological assessment of composite (middling) particles by linear measurements. Proc. $11^{\text {th }}$ Commonwealth
Mining and Metallurgical Congress, p. 113-22.

Jones MP, Coleman R, Horton R (1978). The assessment of composite particles from linear measurements. Proc. $2^{\text {nd }}$ European Symposium on Quantitative Analysis of microstructures, Caen, p. 182.

Lastra R (2002). A comparison of liberation determinations by particle area percentage and exposed particle perimeter percentage in a flotation concentrator. JMMCE 1: 31-7.

Pirard E, Bernhardt HJ, Catalina JC, Brea C, Segundo F, Castroviejo R (2008). From spectrophotometry to multispectral imaging of ore minerals in visible and near infrared (VNIR) microscopy. $9 \mathrm{t}^{\mathrm{h}}$. International Congress for Applied Mineralogy, 2008, Sep 8-10, Brisbane, QLD, Australia, p. 57-62.

Ramdohr P (1980). The ore minerals and their intergrowths, second ed. Oxford: Pergamon Press.

Russ JC (1990), Computer assisted microscopy. New York: Plenum Press.

Russ JC, DeHoff R (2000). Practical stereology. New York: Plenum Press.

Schneider CL (1995). Measurement and calculation of liberation in continuous milling circuits. Ph.D. dissertation. University of Utah.

Underwood EE (1970). Quantitative Stereology. Reading, Massachusetts: Addison Wesley. 